\title{
Management of asymptomatic carotid stenosis
}

\author{
J. David Spence ${ }^{1,2}$
}

${ }^{1}$ Neurology \& Clinical Pharmacology, Western University, London, ON, Canada; ${ }^{2}$ Stroke Prevention \& Atherosclerosis Research Centre, Robarts Research Institute, London, ON, Canada

Correspondence to: J. David Spence, CM, MD, FRCPC, FAHA. Professor of Neurology \& Clinical Pharmacology, Western University; Director, Stroke Prevention \& Atherosclerosis Research Centre, Robarts Research Institute, 1400 Western Road, London, ON, N6G 2V4, Canada.

Email: dspence@robarts.ca.

\begin{abstract}
Patients with asymptomatic carotid stenosis (ACS) are at very high risk of coronary events, so they should all receive intensive medical therapy. What is often accepted as "best medical therapy" is usually suboptimal. Truly intensive medical therapy includes lifestyle modification, particularly smoking cessation and a Mediterranean diet. All patients with ACS should receive intensive lipid-lowering therapy, should have their blood pressure well controlled, and should receive B vitamins for lowering of plasma total homocysteine (tHcy) if levels are high; a commonly missed cause of elevated tHcy is metabolic B12 deficiency, which should be diagnosed and treated. Most patients with ACS would be better treated with intensive medical therapy than with either carotid endarterectomy (CEA) or stenting (CAS). A process called "treating arteries instead of treating risk factors" markedly reduced the risk of ACS in an observational study; a randomized trial vs. usual care should be carried out. The few patients with ACS who could benefit ( 15\%, or perhaps more if recent evidence regarding the risk of intraplaque hemorrhage is borne out) can be identified by a number of features. These include microemboli on transcranial Doppler, intraplaque hemorrhage, reduced cerebrovascular reserve, and echolucency of plaques, particularly "juxtaluminal black plaque". No patient should be subjected to CAS or CEA without evidence of high-risk features, because in most cases the 1-year risk of stroke or death with intervention is higher with either CEA $(\sim 2 \%)$ or CAS $(\sim 4 \%)$ than with intensive medical therapy $(\sim 0.5 \%)$. Most patients, particularly the elderly, would be better treated with CEA than CAS. Most strokes can be prevented in patients with ACS, but truly intensive medical therapy is required.
\end{abstract}

Keywords: Carotid stenosis; endarterectomy; stenting; intensive medical therapy; identifying high-risk asymptomatic stenosis

Submitted Jan 21, 2020. Accepted for publication Apr 27, 2020.

doi: 10.21037/atm-20-975

View this article at: http://dx.doi.org/10.21037/atm-20-975

Patients with asymptomatic carotid stenosis (ACS) are at very high risk of mortality and cardiovascular events; their risk of a myocardial infarction is very high (1), and much higher than their risk of stroke (2). For that reason they should all receive intensive medical therapy, including lifestyle modification. With modern medical therapy the risk of stroke with ACS has declined to $\sim 0.5 \%$ per year, so only a small minority of ACS patients could possibly benefit from carotid endarterectomy (CEA) or stenting (CAS). In this narrative review I discuss medical therapy, ways to identify which patients with ACS could benefit from intervention, and which patients would be more likely to benefit from CEA vs. CAS.

\section{Medical therapy}

Most strokes can be prevented with intensive medical therapy, but in most clinics, what is accepted as "best medical therapy" is suboptimal (3). To maximally reduce the risk of stroke in ACS, medical therapy needs to be truly intensive, as described below, and needs to include lifestyle modification. 


\section{Lifestyle}

Lifestyle is far more important than most physicians suppose. In the Prospective Urban and Rural Epidemiological Study (PURE) study, behavioral risk factors (smoking, alcohol, diet, exercise, and sodium intake) contributed most to deaths (26.3\% of the Population-attributable fractions) (4) In a US study of health professionals $(\mathrm{n}=114,928,61 \%$ male), those who achieved all 5 healthy lifestyle factors (not smoking, moderate alcohol intake, body mass index $<25$, exercise daily 30 minutes, top $40 \%$ healthy diet score) had an $80 \%$ lower risk of stroke (5). Among Swedish men with hypertension and hyperlipidemia, achieving all 5 healthy lifestyle targets reduced coronary events by more than $80 \%$ (6).

\section{Smoking cessation}

Smoking increases the risk of stroke 6-fold, and second hand smoke increases the risk 1.8-fold (7). Among patients with ACS it is therefore imperative that patients stop smoking. They should be persuaded that it does not matter that it is very difficult to quit smoking; it must be done. The parable of the cold lake (borrowed from a Lancet article on weight loss) can be helpful: "If you are walking along the shore of a cold lake and your grandson is drowning, it doesn't take will power to go into the lake; it must be done." Liberal nicotine replacement (as much as it takes to deal with the addiction) should be recommended (8); sometimes varenicline or bupropion can be helpful, but may have adverse effects. The Ottawa protocol for smoking cessation (9) has perhaps been more successful than other approaches. Among patients in the Insulin Resistance Intervention after Stroke (IRIS) trial, smoking cessation was associated with a reduction of the risk of stroke, $M I$ or vascular death by $44 \%$ in 5 years (10).

\section{Diet}

After smoking cessation, the other major opportunity to reduce the risk of stroke is diet. Although it is possible that a vegetarian diet might be even better (if B12 deficiency could be avoided), the best evidence for stroke prevention is for the Cretan Mediterranean Diet. The benefits of that diet were discovered in the Seven Countries Study, in which the coronary risk in Crete was $1 / 15^{\text {th }}$ that in Finland (where $38 \%$ of calories were from animal fat, containing cholesterol and saturated fat), and $2 / 5^{\text {th }}$ that in Japan, where only $10 \%$ of calories were from fat. The Mediterranean diet is high in fat ( $40 \%$ of calories, mainly from olive oil), whole grains, fruits vegetables and legumes. Ancel Keys, the leader of the study, described it as a "a mainly vegetarian diet" (11). As a high-fat diet it is a low glycemic index diet; in an Israeli study it was clearly better for diabetes than either a low-fat diet or a low-carbohydrate diet that was high in cholesterol and saturated fat. In the Lyon Diet Heart Study, the Mediterranean diet was compared to a "prudent Western diet" among persons with coronary artery disease. The Mediterranean diet reduced both stroke and recurrent myocardial infarction by $>60 \%$ in 4 years (12). That was twice the effect of simvastatin in the Scandinavian Simvastatin Survival Study; a $40 \%$ reduction of recurrent myocardial infarction in 6 years (13).

Patients with ACS should keep the intake of animal flesh (mainly fish and chicken, seldom red meat) to $\sim 2$ ounces a day, or a serving the size of their palm about every other day, and avoid egg yolk (14). Egg yolk and red meat are not only high in cholesterol $(180 \mathrm{mg}$ in a 6-ounce serving of meat, and $237 \mathrm{mg}$ in a 63-gram egg); they are also high in dietary precursors of trimethylamine, produced by the intestinal bacteria: carnitine in red meat, and phosphatidylcholine in egg yolk. Trimethylamine is oxidized in the liver to trimethylamine $\mathrm{N}$-oxide (TMAO), which causes atherosclerosis in an animal model (15). Among patients referred for coronary angiography, those with plasma TMAO in the highest quartile had a 2.5 fold increase in the 3-year risk of stroke/MI/vascular death (16). Patients with renal failure have high levels of TMAO, which accelerates decline of renal impairment and increases mortality. Wang et al. reported (17) that switching from red meat to white meat or non-meat protein significantly reduced levels of TMAO within a month. Not only TMAO, but other vasculotoxic metabolites produced by the intestinal microbiome from amino acids in protein, such as p-cresylsulfate, hippuric acid, indoxyl sulfate, p-cresylglucuronide, phenylacetyl glutamine and phenylsulfate, are renally excreted. Even moderate impairment of renal function, to an estimated glomerular filtration rate $(\mathrm{eGFR})<60 \mathrm{~mL} / \mathrm{min} / 1.73 \mathrm{~m}^{2}$ significantly increases their plasma levels (18). Above age 80, the average eGFR is $<60 \mathrm{~mL} / \mathrm{min} / 1.73 \mathrm{~m}^{2}$ (19), so persons with renal impairment, including the elderly, should limit meat intake and avoid egg yolk (14).

\section{Blood pressure control}

Blood pressure control markedly reduces the risk of stroke; it virtually eliminates hypertensive intracerebral 
Table 1 Causes of resistant hypertension

1. Substances ingested by the patient (salt, licorice, decongestants, nonsteroidal anti-inflammatory agents other than sulindac) (25)

2. Non-compliance (about half of patients will admit it if asked in a non-judgemental manner) (26)

3. Therapeutic inertia (failure to initiate or intensify medical therapy)

4. Secondary hypertension (pheochromocytoma, primary aldosteronism, renovascular hypertension, etc.)

5. Diagnostic inertia (failure to investigate the underlying cause of the hypertension) $(27,28)$

Table 2 Physiologically individualized therapy based on renin/aldosterone phenotyping (30)

\begin{tabular}{|c|c|c|c|}
\hline & $\begin{array}{l}\text { Primary aldosteronism/inappropriate } \\
\text { aldosterone secretion }\end{array}$ & $\begin{array}{l}\text { Liddle's syndrome and variants } \\
\text { (mutations affecting the function of the } \\
\text { renal } \mathrm{Na}^{+} \text {channel) }\end{array}$ & Renal/renovascular \\
\hline Aldosterone & High & Low & High \\
\hline $\begin{array}{l}\text { Primary } \\
\text { treatment }\end{array}$ & $\begin{array}{l}\text { Aldosterone antagonist (spironolactone, } \\
\text { eplerenone); amiloride if eplerenone is not } \\
\text { available for } \operatorname{men}^{\dagger} \text { (rarely adrenalectomy) }\end{array}$ & Amiloride & $\begin{array}{l}\text { Angiotensin receptor } \\
\text { blocker, renin inhibitor (rarely } \\
\text { revascularization) }\end{array}$ \\
\hline
\end{tabular}

\footnotetext{
${ }^{\dagger}$, gynecomastia and mastalgia are common in men taking high doses of spironolactone. (Reproduced by permission of Oxford University Press from: Akintunde A, Nondi J, Gogo K, et al. Physiological Phenotyping for Personalized Therapy of Uncontrolled Hypertension in Africa. Am J Hypertens 2017;30:923-30).
}

hemorrhage (20) and markedly reduces the risk of lacunar infarction (21). To achieve control of resistant hypertension, it is important to identify the cause. Among patients with carotid stenosis, renovascular hypertension is common (22), and may require revascularization $(23,24)$. To identify the best medical therapy for patients with resistant hypertension, physiologically individualized therapy based on renin/aldosterone phenotyping (PhysRx) markedly improves blood pressure control. Table 1 lists causes of resistant hypertension; probably the most neglected is "diagnostic inertia"-failure to investigate the underlying cause of the hypertension.

After eliminating rare conditions such as pheochromocytoma and aortic coarctation, hypertension is dominated by the renin/angiotensin/aldosterone axis. Essentially, there are three main groups of hypertensives, and the best medical treatment is different for each of them. Patients with primary aldosteronism/inappropriate aldosterone secretion have a low renin/high aldosterone phenotype, and are best treated with aldosterone antagonists (spironolactone or eplerenone). Those with a low renin/low aldosterone (Liddle) phenotype are best treated with amiloride, and those with a high renin/high aldosterone (Renal) phenotype are best treated with angiotensin receptor blockers or renin inhibitors. Plasma renin and aldosterone should be measured in a stimulated condition (after diuretic, angiotensin receptor antagonist or angiotensin converting enzyme inhibitor), and their values must be interpreted in the light of the class of stimulating drug (29).

The algorithm shown in Table 2 markedly improved blood pressure control in a study in Africa. Among persons with uncontrolled hypertension at the Nigerian site, where conditions were more similar to developed countries than at the other two sites and patients were randomized to PhysRx $v s$. usual care (UC), blood pressure control was much better with PhysRx than with UC. Systolic control was obtained in $15 \%$ of UC vs. $85 \%$ of PhysRx ( $\mathrm{P}=0.0001)$, diastolic control in $45 \%$ vs. $75 \%(\mathrm{P}=0.11)$ and control of both systolic and diastolic pressure in $15 \%$ vs. $75 \%(\mathrm{P}<0.0001)$ even though the patients were older, and renal function was worse at that site. The biggest change in medication was that amiloride was prescribed to $19 \%$ of patients randomized to PhysRx, vs. only $2.8 \%$ on UC (30). Primary aldosteronism and a Liddle phenotype are far commoner than most physicians suppose, because primary aldosteronism is caused by variants of at least 6 genes, and in addition to true Liddle Syndrome (due to variants of SCNN1B, the renal tubular epithelial sodium channel, $\mathrm{ENaC}$ ), there are variants of at least 4 other genes that affect the function of $\mathrm{ENaC}(31,32)$. In the wake of the Systolic Blood Pressure Intervention 
(SPRINT) trial (33), it is important to recognize that patients with stiff arteries and a wide pulse pressure will be put at risk by aiming for a systolic pressure target $<120 \mathrm{mmHg}$ (34). McEvoy et al. (35) reported that patients with a pulse pressure $>60 \mathrm{mmHg}$ and a diastolic pressure $<60 \mathrm{mmHg}(\mathrm{PP}>60 / \mathrm{DBP}<60)$ had a doubling of coronary risk; Park and Ovbiagele reported that they had a 5.85-fold increase in the risk of stroke (36). Reasons for this include: (I) most of coronary perfusion and more than half of cerebral perfusion occur during diastole; (II) there is a large pressure gradient in the brain: "when the blood pressure in the brachial artery is $117 / 75 \mathrm{mmHg}$, it is $113 / 73 \mathrm{mmHg}$ in the lenticulostriate artery but only 59/39 $\mathrm{mmHg}$ in small branches in the posterior parietal subcortex." (37); (III) patients with stiff arteries are more likely to have a large cuff artefact, with the true (intra-arterial) pressure being much lower than the blood pressure measured by a cuff (38).

\section{Lipid lowering therapy}

Patients with ACS are at such a high risk of coronary events that they should all receive intensive lipid-lowering therapy. Adverse effects of statins are much exaggerated, as a result of observational studies with confounding and indication bias, and fallacious attribution of causality (39). Statins do not cause hepatotoxicity, cataracts, cognitive decline, or, absent rhabdomyolysis, renal impairment. In very large randomized trials, the truly causal adverse effects are limited to myopathy and a slight increase in the risk of diabetes. With the exception of a rare autoimmune myopathy, both of these are likely due to depletion of CoQ10 (ubiquinone), and may be remediable with high doses of ubiquinone. Early trials that failed to show benefit of ubiquinone probably used doses that were too low.

An important and neglected way to achieve effective lipid lowering while minimizing adverse effects of statins is to add ezetimibe. Statins block formation of cholesterol, CoQ10 and many other intermediate metabolites in the formation of cholesterol, whereas ezetimibe blocks cholesterol absorption in the lining of the intestine. By blocking two mechanisms the effect is synergistic; $10 \mathrm{mg}$ of atorvastatin with $10 \mathrm{mg}$ of ezetimibe lowers LDL-C by almost as much as $80 \mathrm{mg}$ of atorvastatin, but with less adverse effects. The only truly causal adverse effect of ezetimibe is loose bowel movements; problematic diarrhea is rare.

There is now good evidence that lipid lowering is beneficial in the elderly. Because the elderly are at higher risk of cardiovascular events, they stand to benefit even more than do young people, with a greater absolute risk reduction and a lower number needed to treat (NNT) (40). This issue was recently reviewed (24). In a RCT of adding ezetimibe to simvastatin, the NNT to prevent one cardiovascular event was only 11 over age 75 vs. 125 below age 75 (41). In an RCT in Japan, 3,765 patients with a mean age of 80.7 years at baseline, with risk factors, but free of coronary artery disease, were randomized to diet $v s$. diet plus ezetimibe. Over 5 years, the composite primary endpoint (sudden cardiac death, MI, PCI or CABG, and/or stroke) was reduced by $34 \%$ over 5 years with ezetimibe; there was a $40 \%$ reduction of cardiac events) (42). The recent European Guideline on Dyslipidemias recommends a target LDL-C $<1.4 \mathrm{mmol} / \mathrm{L}$ for patients at very high risk, and recommends addition of ezetimibe in patients who do not achieve target levels with statins as a Grade 1 recommendation (43).

Recent developments in lipid-lowering therapy based on blocking the effects of proprotein convertase subtilisin/ kexin type 9 (PCSK9) are very promising. Injections of antibodies to PCSK9 given once or twice monthly markedly lower LDL-C, with no signal of harm down to LDL-C of $0.2 \mathrm{mmol} / \mathrm{L}$ (44). New approaches are in development. RNA silencing drugs injected once or twice a year are efficacious and appear to be safe (45); regulatory approval is expected soon. However these therapies are very costly. It is to be hoped that small molecules will become available soon and that they would make PCSK9-based therapies affordable.

\section{Antithrombotic therapy}

\section{Antiplatelet agents}

Although there have been recent recommendations that antiplatelet therapy not be used for primary prevention, that would not apply to ACS, since the risk of coronary events in ACS is as high as that in patients with coronary artery disease. There are several issues that deserve discussion in regard to antiplatelet agents. One is the report by Grosser et al. (46), indicating that "aspirin resistance" is probably actually "pseudoresistance", due to enteric coating of aspirin; chewable aspirin may be preferable to coated aspirin.

Another is the issue of dual antiplatelet therapy. Although it is clear from the coronary literature that dual antiplatelet therapy with clopidogrel and aspirin is more effective than either alone, the literature in stroke prevention has tended to recommend only short-term dual therapy, for up to several months. The reason for this has been concern about increased bleeding with longer-term dual therapy, however 
Table 3 Pharmacodynamic and pharmacokinetic properties of direct oral anticoagulants (DOACs)

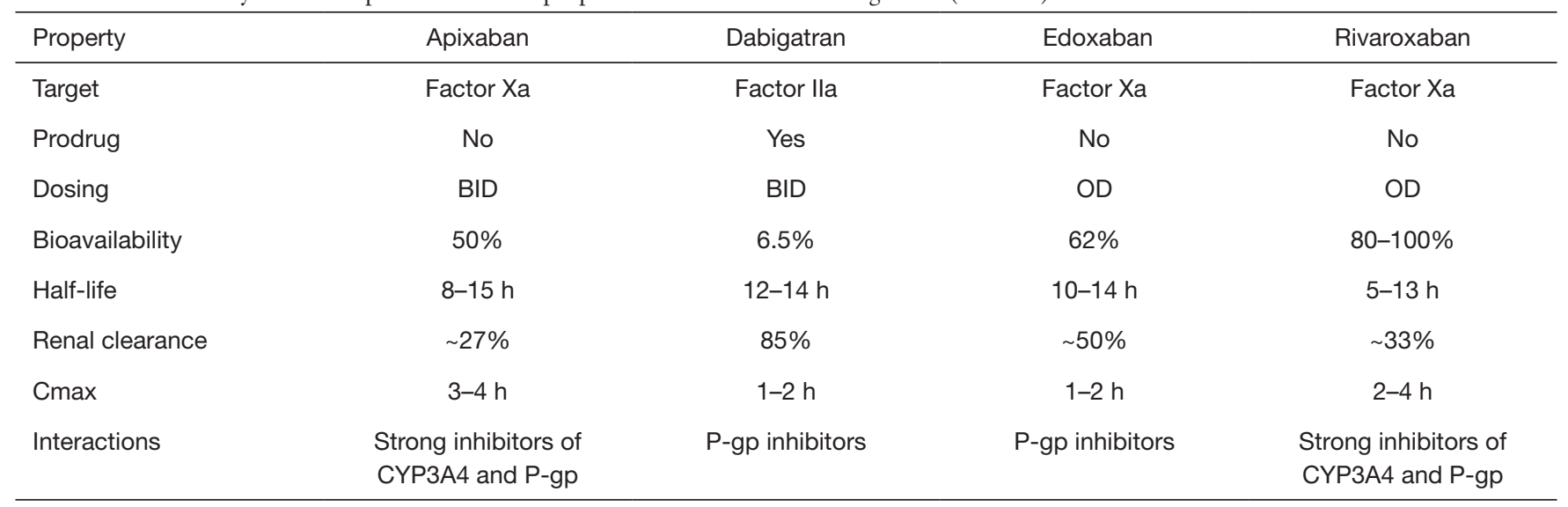

(Reproduced by permission of BMJ from: Spence JD. Cardioembolic stroke: everything has changed. Stroke Vasc Neurol 2018;3:76-83) (51).

BID, twice a day; OD, daily; CMax, time to peak blood level; CYP3A4, cytochrome P4503A4; P-gp, P-glycoprotein.

that concern may be the result of inadequate medical care. Good control of hypertension virtually eliminates hypertensive intracerebral hemorrhage. In the NASCET trial strenuous efforts were made to overcome therapeutic inertia. Every time a patient attended a follow-up visit with a blood pressure above the target and antihypertensive therapy was not intensified, the site investigator received a stiff reminder that the protocol was to be followed. That intensive supervision resulted in a reduction of intracranial hemorrhage to only $0.5 \%$ of strokes (20), at a time when the norm was $20 \%$.

Another important issue for antiplatelet therapy is that clopidogrel is a pro-drug that requires activation by CYP2C19. More than half of Chinese persons, and 30\% of Europeans have variants of that gene that reduce the efficacy of clopidogrel (47). We should be using ticagrelor, or prasugrel. Although prasugrel is a pro-drug, it is activated by esterases (48), so is not affected by variants of CYP2C19 or other cytochrome-based enzymes. Prasugrel was reported to be more efficacious than ticagrelor in acute coronary syndrome (49).

\section{Anticoagulants}

Some patients with ACS may require anticoagulation for cardioembolic sources of stroke, including, but not limited to, atrial fibrillation. In a patient in whom a cardioembolic source is strongly suspected, it is probably prudent to anticoagulate the patient with one of the direct acting anticoagulants (DOACs) (50). It is important to understand the properties of DOACs; though they are all safer than warfarin, and at least two of them have been shown to be no more likely than aspirin to cause severe bleeding, they are not all equally safe and effective within the class. Table 3 highlights important differences.

\section{B vitamins for bomocysteine lowering}

Although it is widely believed that B vitamins to lower plasma total homocysteine (tHcy) do not reduce the risk of stroke, it is now apparent that in the early studies, harm from cyanocobalamin among patients with renal failure obscured the benefit; B vitamins do reduce the risk of stroke, but we should be using methylcobalamin instead of cyanocobalamin (52).

Additional reasons why there was no benefit of $\mathrm{B}$ vitamins in the Vitamin Intervention for Stroke Prevention (VISP) trial were recently reviewed (24). In the French Su.Fol.OM3 trial, in which renal function was the best of the large trials and the dose of cyanocobalamin was much lower than in other trials (20 mcg daily, vs. 400-1,000 mcg daily), the reduction of stroke was $43 \%$ over 4.7 years (53). The China Stroke Primary Prevention Trial (CSPPT) showed clearly that folic acid reduced the risk of stroke in a population where folate fortification is not in place (54); the benefit was proportional to reduction of tHcy (55), and the benefit was greater in higher-risk patients $(56,57)$. Elevated tHcy quadruples the risk of stroke (58), and elevated tHcy is very common in vascular patients: above age $80,40 \%$ of patients had tHcy $>14 \mu \mathrm{mol} / \mathrm{L}(59)$.

A meta-analysis in 2018 concluded that folic acid and $\mathrm{B}$ vitamin combinations reduced the risk of stroke, and 
suggested that in countries with no folate fortification folic acid should be used for stroke prevention (60).

Restricting the recommendation to folic acid is problematic, because in countries with folate fortification, the main nutritional determinant of tHcy is vitamin B12 deficiency $(61,62)$. Both biochemical B12 deficiency (a serum B12 below the reference range) and metabolic B12 deficiency (serum B12 below the median and tHcy $>14 \mu \mathrm{mol} / \mathrm{L})$ are common. Before 2009, when B12 supplementation increased in patients referred with stroke/TIA, biochemical B12 deficiency was present in $10 \%$ of patients referred to an Urgent TIA Clinic (63), and metabolic B12 deficiency was present in $30 \%$ of patients over age 71 (64). After 2009, with increased B12 supplementation in our region, biochemical B12 deficiency declined to $5 \%$ of patients, but at age $70-79,10.9 \%$ of patients had metabolic B12 deficiency and at age $>80$ years it was $15.1 \%$. It is likely that this is more common in other jurisdictions. In the Newcastle $85+$ study, at least $25 \%$ of patients had metabolic B12 deficiency. Because a serum $\mathrm{B} 12$ in the reference range does not identify metabolic B12 deficiency, it is often/usually missed, so it represents an important missed opportunity to prevent stroke and dementia (65).

It might be thought that in countries with folate fortification, only vitamin B12 would be needed for stroke prevention; however two recent studies indicate that patients with the $\mathrm{T}$ allele of methylenetetrahydrofolate reductase (MTHFR) require higher doses of folate than would be obtained from folate fortification $(66,67)$. This is important because the $\mathrm{T}$ allele, which is associated with higher levels of tHcy is common. In the CSPPT the prevalence of genotypes in Chinese participants was $27 \%$ CC, $49 \%$ CT, and 24\% TT. In a mainly European population of patients referred for stroke prevention, the prevalence was $40.4 \% \mathrm{CC}, 46.6 \% \mathrm{CT}$, and $13 \% \mathrm{TT}$. Two recent studies indicate that higher doses of folate are required for patients with the $\mathrm{T}$ allele $(66,67)$. It is therefore important to recognize that even in the presence of folate fortification, a substantial proportion of patients would require additional folate supplementation.

\section{How to identify which patients with ACS could benefit from intervention}

As discussed above, most patients with ACS would be better treated with intensive medical therapy than with CEA or CAS. However, it is probable that $\sim 15 \%$ could benefit from intervention. That estimate may need to be revised upward in view of recent evidence regarding intraplaque hemorrhage, as discussed below. They can be identified by a number of features. Perhaps the best validated is transcranial Doppler microembolus detection (Figure 1). Patients with ACS and 2 or more microemboli in one hour of monitoring had a 1 -year risk of stroke of $15.6 \%$, vs. $1 \%$ without microemboli (68). Those with 1 or more microemboli had an annual risk of ipsilateral stroke or TIA over 2 years of $7.13 \%$ with microemboli vs. $3.04 \%$ without microemboli. Patients with 3 or more carotid ulcers (on either or both sides) have a stroke risk similar to that of microemboli (69), and ulcers identify a different population of high-risk ACS. Paraskevas et al. reviewed a number of clinical and imaging features of high-risk ACS that are accepted by the European Society of Vascular Surgery (70) as indications for intervention in ACS. In addition to microemboli on transcranial Doppler (TCD), they include progression of severe stenosis, reduced cerebrovascular reserve, intraplaque hemorrhage, echolucent plaque and "juxtaluminal black plaque" (71) (Table 4).

A meta-analysis in 2019 reported that $29.4 \%$ of patients with ACS had intraplaque hemorrhage on MRI, and the annualized risk of ipsilateral stroke with intraplaque hemorrhage was " $9.0 \%$ versus $0.7 \%$ ( $<50 \%$ stenosis), $18.1 \%$ versus $2.1 \%$ (50\% to $69 \%$ stenosis), and $29.3 \%$ versus $1.5 \%$ (70\% to $99 \%$ stenosis) (72). However, that study included only 126 patients with ACS, so further study is needed, including assessment of the size of intraplaque hemorrhage.

\section{"Treating arteries instead of treating risk factors"}

In 2002 we reported that carotid plaque burden, measured by ultrasound as total plaque area (TPA) was a very strong predictor of cardiovascular risk. By quartile of TPA, the 5 -year risk of stroke/MI/vascular death was $5.6 \%, 10.7 \%$, $13.9 \%$, and $19.5 \%$, after adjustment for age, sex, systolic blood pressure, smoking (pack-years), diabetes, serum total cholesterol, tHcy and treatment of blood pressure and cholesterol (73). Carotid plaque burden is highly correlated with coronary calcium score (74), and as predictive of cardiovascular events (75). In the first year of follow-up, half the patients had progression of plaque despite usual therapy, and those with plaque progression had twice the risk of those events, after adjustment for those risk factors (73). This meant that usual therapy was failing half our patients; we needed a better way of doing things. In 2003 we 


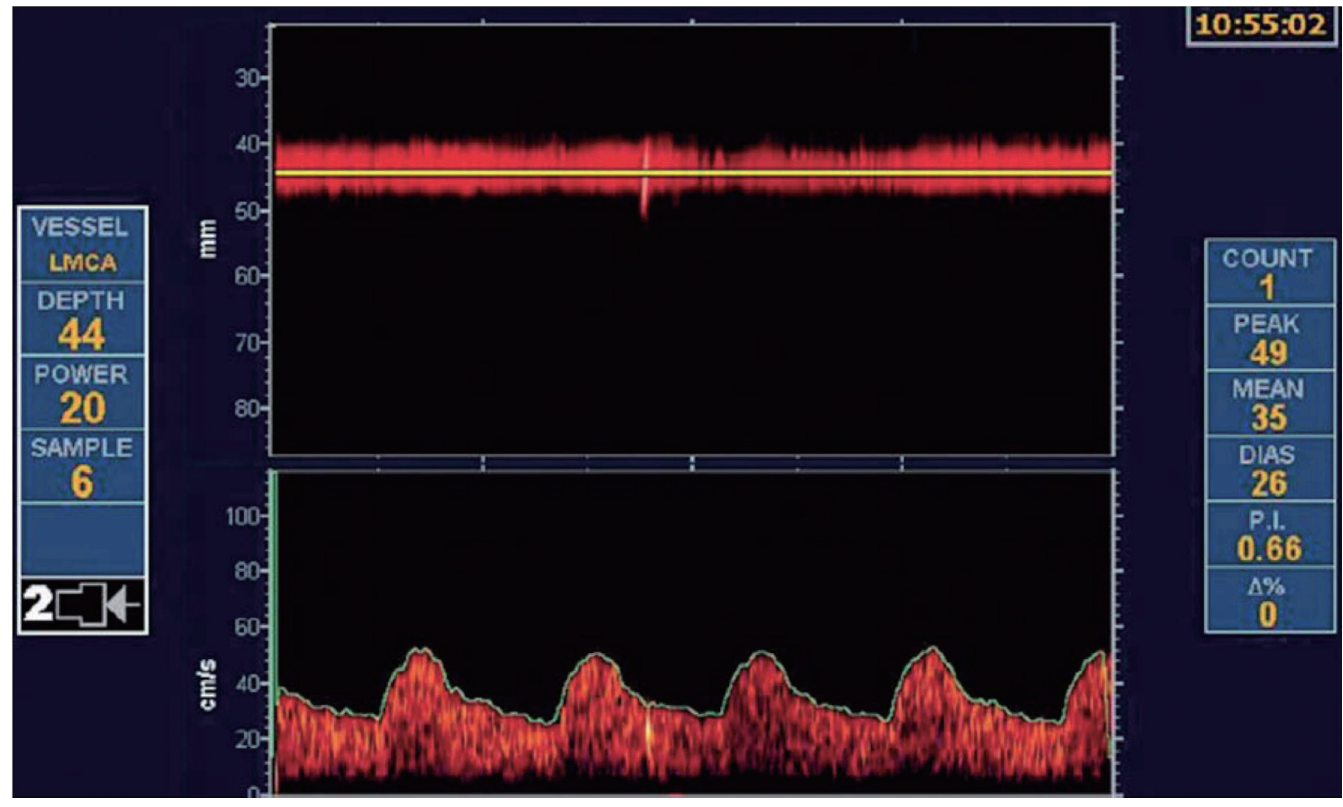

Figure 1 Transcranial Doppler embolus detection. Microembolus in a patient with asymptomatic carotid stenosis. The upper channel is an M-mode image of an embolus in the middle cerebral artery; the lower panel shows the high-intensity transit signal in the Doppler channel. Besides the visual appearance of the microembolus, a characteristic clicking sound is heard. (Reproduced by permission of the Society for Vascular Ultrasound from: Spence JD. Transcranial Doppler: uses in stroke prevention. The fournal for Vascular Ultrasound 2015;39:183-7).

Table 4 Characteristics of high-risk patients with asymptomatic carotid stenosis

\begin{tabular}{lc}
\hline Imaging/clinical parameter & OR/HR (95\% Cl), P value \\
\hline Spontaneous embolization on TCD & $7.46(2.24-24.89), 0.001$ \\
Plaque echolucency on Duplex US & $2.61(2.98-4.63), 0.001$ \\
Spontaneous emboli on TCD + echolucency & $10.61(2.98-37.82), 0.0003$ \\
Progression of stenosis (50-99\% stenoses) & $1.92(1.14-3.25), 0.05$ \\
Progression of stenosis (70-99\% stenoses) & $4.7(2.3-9.6), 0.01$ \\
Silent infarction on CT (60-99\% stenoses) & $3.0(1.46-6.29), 0.002$ \\
Impaired CBF reserve (70-99\% stenoses) & $6.14(2.77-4.95),<0.01$ \\
Juxtaluminal black plaque area (<4, 4-8, 8-10, >10 mm $\left.{ }^{2}\right)$ & $\mathrm{P}$ for trend <0.001 \\
Intraplaque hemorrhage on MRI & $3.66(2.77-4.95),<0.01$ \\
Contralateral stroke/TIA & $3.0(1.9-4.73), 0.0001$ \\
\hline
\end{tabular}

(Reproduced by permission of BMJ from: Naylor AR, Ricco JB, de Borst GJ, et al. Editor's Choice - Management of Atherosclerotic Carotid and Vertebral Artery Disease: 2017 Clinical Practice Guidelines of the European Society for Vascular Surgery (ESVS). Eur $J$ Vasc Endovasc Surg 2018;55:3-81). TCD, transcranial Doppler; TIA, transient ischemic attack; US, ultrasound; MRI, magnetic resonance imaging.

implemented in our clinics a paradigm change, "Treating arteries instead of treating risk factors" (76). This is feasible because carotid plaque progresses and regresses much faster than most would think (77) (Figure 2). It could not be done with carotid intima-media thickness, coronary calcium, or intracoronary vascular ultrasound, because they do not change in response to therapy in a clinically meaningful time scale $(77,78)$. 


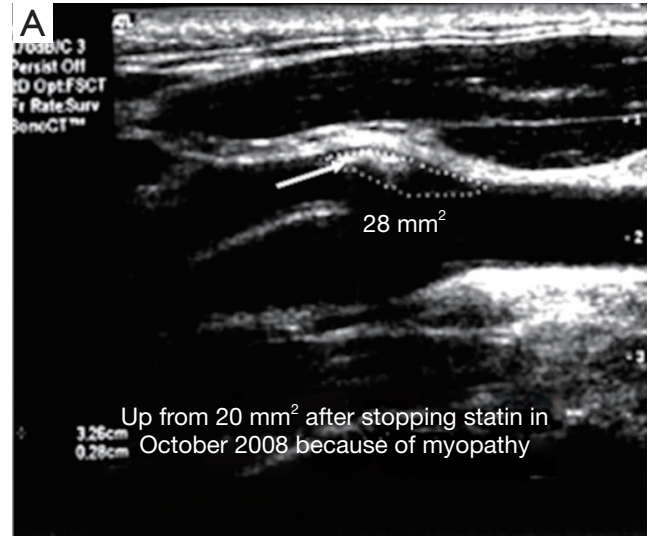

April 15, 2009

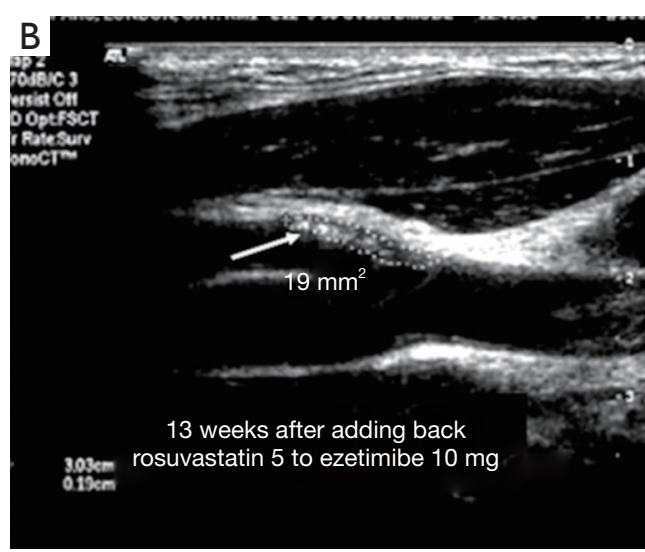

July 23, 2009

Figure 2 Plaque regression is much faster than most would expect. (A) Soft plaque at the origin of the left external carotid in a 64-year-old man using ezetimibe alone because of myalgia and cramps with statins. His plaque (white arrow) had progressed from $20 \mathrm{~mm}^{2} 6 \mathrm{months}$ earlier, to $28 \mathrm{~mm}^{2}$ after stopping rosuvastatin and taking ezetimibe alone. After restarting rosuvastatin $5 \mathrm{mg}$ daily with ezetimibe $10 \mathrm{mg}$ daily, and CoQ10 $200 \mathrm{mg}$ daily to prevent myalgia, the plaque area regressed to $19 \mathrm{~mm}^{2}$ over 13 weeks (B). The plaque had also become denser, with regression of the echolucent plaque. (Reproduced by permission of Elsevier from: Spence JD. Coronary calcium is not all we need: Carotid plaque burden measured by ultrasound is better. Atherosclerosis 2019;287:179-80.).

The goal of therapy is not simply to achieve target levels of risk factors such as LDL-C and blood pressure; it is to stop progression or achieve regression of plaque. The elements of "Treating Arteries instead of treating risk factors" are shown in Table 5 (80). A key aspect of the process is that showing patients images of their arteries markedly improves compliance with medical advice $(81,82)$.

By 2010 we had evidence that this was extremely effective in patients with ACS: the percent of patients with TCD microemboli declined from $12.6 \%$ to $3.7 \%$, the rate of progression of carotid plaque declined significantly, and more importantly, there was a $>80 \%$ reduction in the 2 -year risk of stroke or myocardial infarction (83).

"Treating atherosclerosis without measuring plaque would be like treating hypertension without measuring the blood pressure." (76). A randomized trial of "treating arteries" vs. usual care should be carried out.

\section{Choosing CEA vs. CAS}

On average, the risk of stroke is higher with CAS than with CEA. A meta-analysis reported that "the periprocedural and postprocedural risks combined favoured CEA, with treatment differences at 1, 3, 5, 7, and 9 years all ranging between $2.8 \%(1.1-4.4 \%)$ and $4.1 \%(2.0-6.3 \%) "(84)$. Most patients with ACS would be better treated with CEA than with CAS. This is particularly the case for older patients. A meta-analysis reported that compared with patients younger than 60 years, the hazard ratio for stroke and death with CAS was 2.16 at age $65-69$, and 4.0 above age 70 (85). This probably relates to a higher risk of emboli during stenting in patients with tortuous, stiff craggy arteries. Figure 3 shows microemboli during stenting of carotid stenosis (86). Almekhlafi et al. reported that $80 \%$ of patients had new infarctions (shown as diffusion restriction lesions on MRI) after carotid stenting, and $6.7 \%$ had worsening of their clinical status (87).

Patients in whom CAS might be preferred to CAS would include younger patients, and those with specific anatomical features such as distal stenosis, lack of tortuosity of the arteries, absent or minimal plaque calcification, local tissue scarring due to previous surgery or radiation, and those with a high medical risk for surgery (88).

\section{Conclusions}

Patients with ACS are at very high risk of vascular events, so they should all receive truly intensive medical therapy, including risk factor modification. Only a small minority could benefit from CEA or CAS; most would be better treated medically. The few who could benefit can be identified by a number of high-risk features. Of those, most would be better treated with endarterectomy than with stenting. 
Table 5 Elements of "Treating arteries instead of treating risk factors"

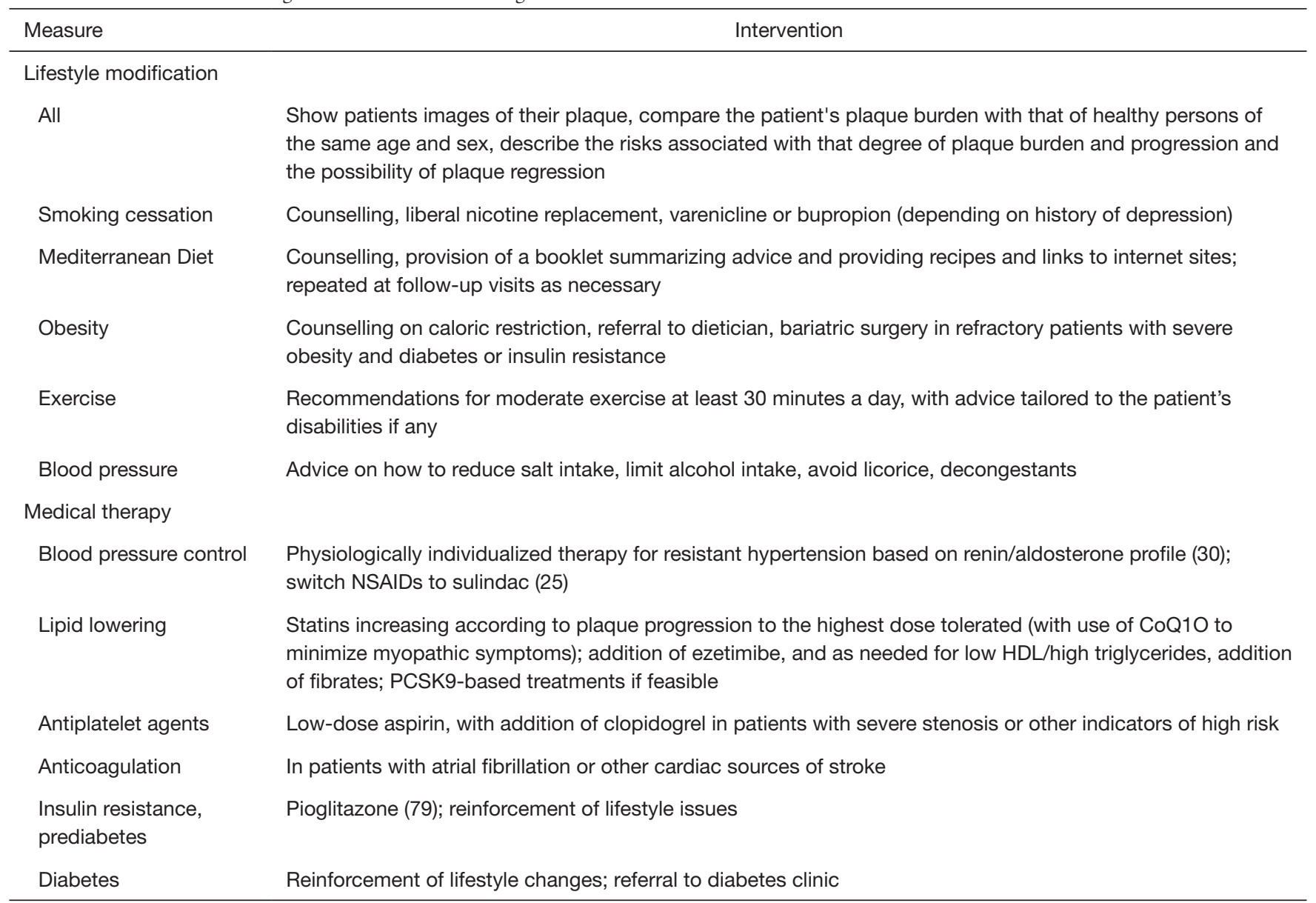

Reproduced by permission of the American Heart Association (with updating of references) from the supplement to: Yang C, Bogiatzi C, Spence JD. Risk of Stroke at the Time of Carotid Occlusion. JAMA Neurol 2015;72:1261-7.
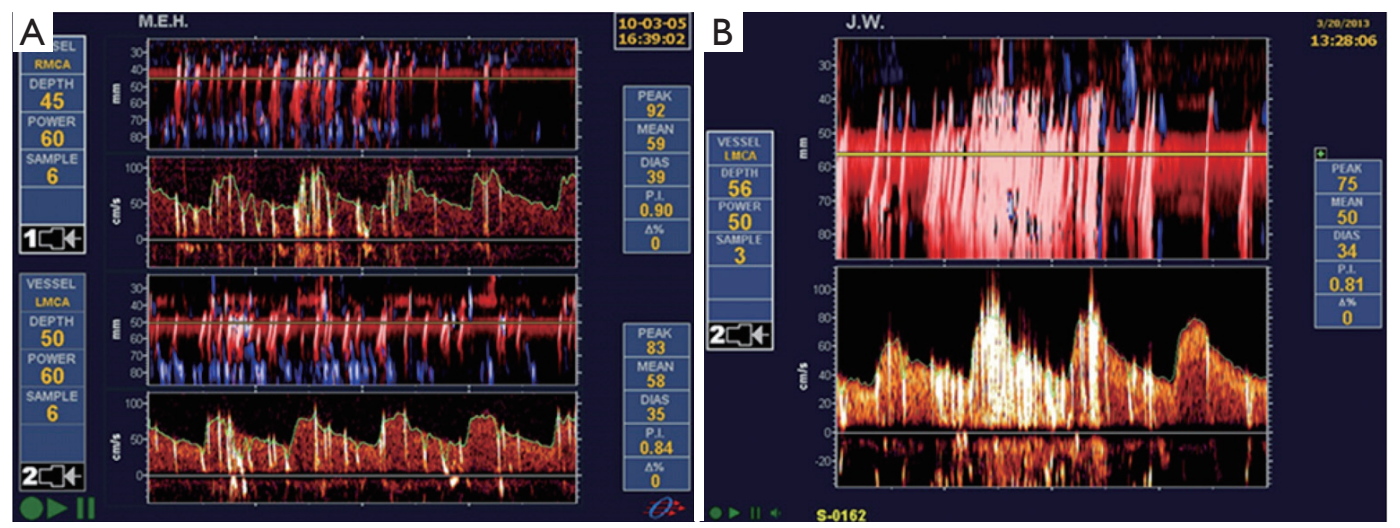

Figure 3 Microemboli during carotid stenting. Showers of emboli commonly (even usually) occur during carotid stenting. (A) shows microemboli while crossing the aortic arch during stenting of a common carotid; (B) shows microemboli during stenting of an internal carotid artery. (Courtesy of Dr. Claudio Muñoz). (Reproduced by permission of Springer from: Spence JD. Management of Patients with an Asymptomatic Carotid Stenosis-Medical Management, Endovascular Treatment, or Carotid Endarterectomy? Curr Neurol Neurosci Rep 2016;16:3). 


\section{Acknowledgments}

Funding: None.

\section{Footnote}

Provenance and Peer Review: This article was commissioned by the Guest Editor (Dr. Kosmas I. Paraskevas) for the series "Carotid Artery Stenosis and Stroke: Prevention and Treatment Part I" published in Annals of Translational Medicine. The article was sent for external peer review organized by the Guest Editor and the editorial office.

Conflicts of Interest: The author has completed the ICMJE uniform disclosure form (available at http://dx.doi. org/10.21037/atm-20-975). The series "Carotid Artery Stenosis and Stroke: Prevention and Treatment Part I" was commissioned by the editorial office without any funding or sponsorship. JDS reports personal fees from Amgen, personal fees from Orphan Technologies, other from Vascularis Inc., outside the submitted work. In addition, JDS has a small share of a patent on measurement of carotid plaque by 3D ultrasound, with royalties paid by Enable Imaging Inc.

Etbical Statement: The author is accountable for all aspects of the work in ensuring that questions related to the accuracy or integrity of any part of the work are appropriately investigated and resolved.

Open Access Statement: This is an Open Access article distributed in accordance with the Creative Commons Attribution-NonCommercial-NoDerivs 4.0 International License (CC BY-NC-ND 4.0), which permits the noncommercial replication and distribution of the article with the strict proviso that no changes or edits are made and the original work is properly cited (including links to both the formal publication through the relevant DOI and the license). See: https://creativecommons.org/licenses/by-nc-nd/4.0/.

\section{References}

1. Chimowitz MI, Weiss DG, Cohen SL, et al. Cardiac prognosis of patients with carotid stenosis and no history of coronary artery disease. Veterans Affairs Cooperative Study Group 167. Stroke 1994;25:759-65.

2. Norris JW, Zhu CZ, Bornstein NM, et al. Vascular risks of asymptomatic carotid stenosis. Stroke 1991;22:1485-90.
3. Spence JD. Asymptomatic Carotid Stenosis: High Risk With Suboptimal Treatment: Re: Five Year Outcomes in Men Screened for Carotid Artery Stenosis at 65 Years of Age: A Population Based Cohort Study. Eur J Vasc Endovasc Surg 2020;59:151.

4. Yusuf S, Joseph P, Rangarajan S, et al. Modifiable risk factors, cardiovascular disease, and mortality in 155722 individuals from 21 high-income, middle-income, and low-income countries (PURE): a prospective cohort study. Lancet 2020;395:795-808.

5. Chiuve SE, Rexrode KM, Spiegelman D, et al. Primary prevention of stroke by healthy lifestyle. Circulation 2008;118:947-54.

6. Akesson A, Larsson SC, Discacciati A, et al. Low-risk diet and lifestyle habits in the primary prevention of myocardial infarction in men: a population-based prospective cohort study. J Am Coll Cardiol 2014;64:1299-306.

7. Bonita R, Duncan J, Truelsen R, et al. Passive smoking as well as active smoking increases the risk of acute stroke. Tobacco Control 1999;8:156-60.

8. Pipe A. Smoking Cessation. In: Spence JD, Barnett HJ, editors. Stroke Prevention, Treatment and Rehabilitation. New York: McGraw-Hill Medical; 2012.

9. Reid RD, Mullen KA, Slovinec D'Angelo ME, et al. Smoking cessation for hospitalized smokers: an evaluation of the "Ottawa Model". Nicotine Tob Res 2010;12:11-8.

10. Epstein KA, Viscoli CM, Spence JD, et al. Smoking cessation and outcome after ischemic stroke or TIA. Neurology 2017;89:1723-9.

11. Keys A. Mediterranean diet and public health: personal reflections. Am J Clin Nutr 1995;61:1321S-3S.

12. Renaud S, de Lorgeril M, Delaye J, et al. Cretan Mediterranean diet for prevention of coronary heart disease. Am J Clin Nutr 1995;61:1360S-7S.

13. Randomised trial of cholesterol lowering in 4444 patients with coronary heart disease: the Scandinavian Simvastatin Survival Study (4S). Lancet 1994;344:1383-9.

14. Spence JD. Diet for stroke prevention. Stroke Vasc Neurol 2018;3:44-50.

15. Wang Z, Klipfell E, Bennett BJ, et al. Gut flora metabolism of phosphatidylcholine promotes cardiovascular disease. Nature 2011;472:57-63.

16. Tang WHW, Wang Z, Levinson BS, et al. Intestinal Microbiota Metabolism of Phosphatidylcholine and Cardiovascular Risk. N Engl J Med 2013;368:1575-84.

17. Wang Z, Bergeron N, Levison BS, et al. Impact of chronic dietary red meat, white meat, or non-meat protein on trimethylamine $\mathrm{N}$-oxide metabolism and renal excretion in 
healthy men and women. Eur Heart J 2019;40:583-94.

18. Pignanelli M, Bogiatzi C, Gloor G, et al. Moderate Renal Impairment and Toxic Metabolites Produced by the Intestinal Microbiome: Dietary Implications. J Ren Nutr 2019;29:55-64.

19. Spence JD, Urquhart BL, Bang H. Effect of renal impairment on atherosclerosis: only partially mediated by homocysteine. Nephrol Dial Transplant 2016;31:937-44.

20. Barnett HJM, Taylor DW, Eliasziw M, et al. Benefit of carotid endarterectomy in patients with symptomatic moderate or severe carotid stenosis. N Engl J Med 1998;339:1415-25.

21. Spence JD. Antihypertensive drugs and prevention of atherosclerotic stroke. Stroke 1986;17:808-10.

22. Spence JD. Management of resistant hypertension in patients with carotid stenosis: High prevalence of renovascular hypertension. Cerebrovasc Dis 2000;10:249-54.

23. Spence JD. Treatment options for renovascular hypertension. Expert Opin Pharmacother 2002;3:411-6.

24. Spence JD. The need for clinical judgement in the application of evidence-based medicine. BMJ Evid Based Med 2019. [Epub ahead of print].

25. Wong DG, Spence JD, Lamki L, et al. Effect of nonsteroidal anti-inflammatory drugs on control of hypertension by beta-blockers and diuretics. Lancet 1986;1:997-1001.

26. Haynes RB, Taylor DW, Sackett DL, et al. Can simple clinical measurements detect patient noncompliance? Hypertension 1980;2:757-64.

27. Spence JD, Rayner BL. J Curve and Cuff Artefact, and Diagnostic Inertia in Resistant Hypertension. Hypertension 2016;67:32-3.

28. Spence JD. Controlling resistant hypertension. Stroke Vasc Neurol 2018;3:69-75.

29. Huang X, Li J, Liu L, et al. Interpreting stimulated plasma renin and aldosterone to select physiologically individualized therapy for resistant hypertension: importance of the class of stimulating drugs. Hypertens Res 2019;42:1971-8.

30. Akintunde A, Nondi J, Gogo K, et al. Physiological Phenotyping for Personalized Therapy of Uncontrolled Hypertension in Africa. Am J Hypertens 2017;30:923-30.

31. Spence JD, Rayner BL. Hypertension in Blacks: Individualized Therapy Based on Renin/Aldosterone Phenotyping. Hypertension 2018;72:263-9.

32. Spence JD. Hypertension in Africa. Eur J Prev Cardiol 2019;26:455-7.
33. SPRINT Research Group, Wright JT Jr, Williamson JD, et al. A Randomized Trial of Intensive versus Standard Blood-Pressure Control. N Engl J Med 2015;373:2103-16.

34. Spence JD. Systolic blood pressure targets, diastolic J curve and cuff artefact in blood pressure measurement: a note of caution. Eur J Neurol 2017;24:1323-5.

35. McEvoy JW, Chen Y, Rawlings A, et al. Diastolic Blood Pressure, Subclinical Myocardial Damage, and Cardiac Events: Implications for Blood Pressure Control. J Am Coll Cardiol 2016;68:1713-22.

36. Park JH, Ovbiagele B. Post-stroke diastolic blood pressure and risk of recurrent vascular events. Eur J Neurol 2017;24:1416-23.

37. Blanco PJ, Muller LO, Spence JD. Blood pressure gradients in cerebral arteries: a clue to pathogenesis of cerebral small vessel disease. Stroke Vasc Neurol 2017;2:108-17.

38. Finnegan TP, Spence JD, Wong DG, et al. Blood pressure measurement in the elderly: correlation of arterial stiffness with difference between intra-arterial and cuff pressures. J Hypertens 1985;3:231-5.

39. Collins R, Reith C, Emberson J, et al. Interpretation of the evidence for the efficacy and safety of statin therapy. Lancet 2016;388:2532-61.

40. Mortensen MB, Falk E. Primary Prevention With Statins in the Elderly. J Am Coll Cardiol 2018;71:85-94.

41. Gotto AM Jr. Intensive Lipid Lowering in Elderly Patients. JAMA Cardiol 2019. [Epub ahead of print].

42. Ouchi Y, Sasaki J, Arai H, et al. Ezetimibe Lipid-Lowering Trial on Prevention of Atherosclerotic Cardiovascular Disease in 75 or Older (EWTOPIA 75): A Randomized, Controlled Trial. Circulation 2019;140:992-1003.

43. Mach F, Baigent C, Catapano AL, et al. 2019 ESC/EAS Guidelines for the management of dyslipidaemias: lipid modification to reduce cardiovascular risk. Eur Heart J 2020;41:111-88.

44. Giugliano RP, Wiviott SD, Blazing MA, et al. Long-term safety and efficacy of achieving very low levels of lowdensity lipoprotein cholesterol: A prespecified analysis of the improve-it trial. JAMA Cardiol 2017;2:547-55.

45. Ray KK, Stoekenbroek RM, Kallend D, et al. Effect of 1 or 2 Doses of Inclisiran on Low-Density Lipoprotein Cholesterol Levels: One-Year Follow-up of the ORION-1 Randomized Clinical Trial. JAMA Cardiol 2019. [Epub ahead of print].

46. Grosser T, Fries S, Lawson JA, et al. Drug resistance and pseudoresistance: an unintended consequence of enteric coating aspirin. Circulation 2013;127:377-85. 
47. Wang Y, Zhao X, Lin J, et al. Association Between CYP2C19 Loss-of-Function Allele Status and Efficacy of Clopidogrel for Risk Reduction Among Patients With Minor Stroke or Transient Ischemic Attack. JAMA 2016;316:70-8.

48. Schömig A. Ticagrelor--is there need for a new player in the antiplatelet-therapy field? N Engl J Med 2009;361:1108-11.

49. Schüpke S, Neumann FJ, Menichelli M, et al. Ticagrelor or Prasugrel in Patients with Acute Coronary Syndromes. N Engl J Med 2019;381:1524-34.

50. Spence JD. Anticoagulation in patients with Embolic Stroke of Unknown Source. Int J Stroke 2019;14:334-6.

51. Spence JD. Cardioembolic stroke: everything has changed. Stroke Vasc Neurol 2018;3:76-83.

52. Spence JD, Yi Q, Hankey GJ. B vitamins in stroke prevention: time to reconsider. Lancet Neurol 2017;16:750-60.

53. Galan P, Kesse-Guyot E, Czernichow S, et al. Effects of $\mathrm{B}$ vitamins and omega 3 fatty acids on cardiovascular diseases: a randomised placebo controlled trial. BMJ 2010;341:c6273.

54. Huo Y, Li J, Qin X, et al. Efficacy of folic acid therapy in primary prevention of stroke among adults with hypertension in China: the CSPPT randomized clinical trial. JAMA 2015;313:1325-35.

55. Huang X, Li Y, Li P, et al. Association between percent decline in serum total homocysteine and risk of first stroke. Neurology 2017;89:2101-7.

56. Qin X, Li J, Spence JD, et al. Folic Acid Therapy Reduces the First Stroke Risk Associated With Hypercholesterolemia Among Hypertensive Patients. Stroke 2016;47:2805-12.

57. Kong X, Huang X, Zhao M, et al. Platelet Count Affects Efficacy of Folic Acid in Preventing First Stroke. J Am Coll Cardiol 2018;71:2136-46.

58. Poli D, Antonucci E, Cecchi E, et al. Culprit factors for the failure of well-conducted warfarin therapy to prevent ischemic events in patients with atrial fibrillation: the role of homocysteine. Stroke 2005;36:2159-63.

59. Spence D. Mechanisms of thrombogenesis in atrial fibrillation. Lancet 2009;373:1006-7.

60. Jenkins DJA, Spence JD, Giovannucci EL, et al. Supplemental Vitamins and Minerals for CVD Prevention and Treatment. J Am Coll Cardiol 2018;71:2570-84.

61. Quinlivan EP, McPartlin J, McNulty H, et al. Importance of both folic acid and vitamin B12 in reduction of risk of vascular disease. Lancet 2002;359:227-8.
62. Robertson J, Iemolo F, Stabler SP, et al. Vitamin B12, homocysteine and carotid plaque in the era of folic acid fortification of enriched cereal grain products. CMAJ 2005;172:1569-73.

63. Ahmed S, Bogiatzi C, Hackam DG, et al. Vitamin B 12 deficiency and hyperhomocysteinaemia in outpatients with stroke or transient ischaemic attack: a cohort study at an academic medical centre. BMJ Open 2019;9:e026564.

64. Spence JD. Nutrition and stroke prevention. Stroke 2006;37:2430-5.

65. Spence JD. Metabolic vitamin B12 deficiency: a missed opportunity to prevent dementia and stroke. Nutr Res 2016;36:109-16.

66. Li Y, Spence JD, Wang X, et al. Effect of Vitamin B12 Levels on the Association Between Folic Acid Treatment and CKD Progression: A Post Hoc Analysis of a Folic Acid Interventional Trial. Am J Kidney Dis 2020;75:325-32.

67. Qin X, Spence JD, Li J, et al. Interaction of serum vitamin B12 and folate with MTHFR genotypes on risk of ischemic stroke. Neurology 2020. [Epub ahead of print].

68. Spence JD, Tamayo A, Lownie SP, et al. Absence of microemboli on transcranial Doppler identifies lowrisk patients with asymptomatic carotid stenosis. Stroke 2005;36:2373-8.

69. Madani A, Beletsky V, Tamayo A, et al. High-risk asymptomatic carotid stenosis: ulceration on $3 \mathrm{D}$ ultrasound vs TCD microemboli. Neurology 2011;77:744-50.

70. Naylor AR, Ricco JB, de Borst GJ, et al. Editor's Choice - Management of Atherosclerotic Carotid and Vertebral Artery Disease: 2017 Clinical Practice Guidelines of the European Society for Vascular Surgery (ESVS). Eur J Vasc Endovasc Surg 2018;55:3-81.

71. Paraskevas KI, Veith FJ, Spence JD. How to identify which patients with asymptomatic carotid stenosis could benefit from endarterectomy or stenting. Stroke Vasc Neurol 2018;3:92-100.

72. Schindler A, Schinner R, Altaf N, et al. Prediction of Stroke Risk by Detection of Hemorrhage in Carotid Plaques: Meta-Analysis of Individual Patient Data. JACC Cardiovasc Imaging 2020;13:395-406.

73. Spence JD, Eliasziw M, DiCicco M, et al. Carotid plaque area: a tool for targeting and evaluating vascular preventive therapy. Stroke 2002;33:2916-22.

74. Sillesen H, Muntendam P, Adourian A, et al. Carotid Plaque Burden as a Measure of Subclinical Atherosclerosis: Comparison With Other Tests for Subclinical Arterial Disease in the High Risk Plaque BioImage Study. JACC Cardiovasc Imaging 2012;5:681-9. 
75. Baber U, Mehran R, Sartori S, et al. Prevalence, impact, and predictive value of detecting subclinical coronary and carotid atherosclerosis in asymptomatic adults: the BioImage study. J Am Coll Cardiol 2015;65:1065-74.

76. Spence JD, Hackam DG. Treating arteries instead of risk factors: a paradigm change in management of atherosclerosis. Stroke 2010;41:1193-9.

77. Spence JD. Time course of atherosclerosis regression. Atherosclerosis 2014;235:347-8.

78. Spence JD. Coronary calcium is not all we need: Carotid plaque burden measured by ultrasound is better. Atherosclerosis 2019;287:179-80.

79. Spence JD, Viscoli CM, Inzucchi SE, et al. Pioglitazone Therapy in Patients With Stroke and Prediabetes: A Post Hoc Analysis of the IRIS Randomized Clinical Trial. JAMA Neurol 2019;76:526-35.

80. Yang C, Bogiatzi C, Spence JD. Risk of Stroke at the Time of Carotid Occlusion. JAMA Neurol 2015;72:1261-7.

81. Korcarz CE, DeCara JM, Hirsch AT, et al. Ultrasound detection of increased carotid intima-media thickness and carotid plaque in an office practice setting: does it affect physician behavior or patient motivation? J Am Soc Echocardiogr 2008;21:1156-62.

82. Näslund U, $\mathrm{Ng} \mathrm{N}$, Lundgren A, et al. Visualization of asymptomatic atherosclerotic disease for optimum

Cite this article as: Spence JD. Management of asymptomatic carotid stenosis. Ann Transl Med 2020;8(19):1262. doi: 10.21037/atm-20-975 cardiovascular prevention (VIPVIZA): a pragmatic, openlabel, randomised controlled trial. Lancet 2019;393:133-42.

83. Spence JD, Coates V, Li H, et al. Effects of intensive medical therapy on microemboli and cardiovascular risk in asymptomatic carotid stenosis. Arch Neurol 2010;67:180-6.

84. Brott TG, Calvet D, Howard G, et al. Long-term outcomes of stenting and endarterectomy for symptomatic carotid stenosis: a preplanned pooled analysis of individual patient data. Lancet Neurol 2019;18:348-56.

85. Howard G, Roubin GS, Jansen O, et al. Association between age and risk of stroke or death from carotid endarterectomy and carotid stenting: a meta-analysis of pooled patient data from four randomised trials. Lancet 2016;387:1305-11.

86. Spence JD. Management of Patients with an Asymptomatic Carotid Stenosis-Medical Management, Endovascular Treatment, or Carotid Endarterectomy? Curr Neurol Neurosci Rep 2016;16:3.

87. Almekhlafi MA, Demchuk AM, Mishra S, et al. Malignant emboli on transcranial Doppler during carotid stenting predict postprocedure diffusion-weighted imaging lesions. Stroke 2013;44:1317-22.

88. Spence JD. Periprocedural events dominate outcomes of carotid stenting and endarterectomy. Lancet Neurol 2019;18:321-2. 